Anaesthesist 2010 59 :505-506 DOI 10.1007/s00101-010-1744-4 Online publiziert: 11. Juni 2010

(c) Springer-Verlag 2010
P. Tassani-Prell ${ }^{1}$ G. Juchem ${ }^{2}$

${ }^{1}$ Institut für Anästhesiologie, Deutsches Herzzentrum München, Klinik für Anaesthesiologie der Technischen Universität Klinikum rechts der Isar, München

${ }^{2}$ Herzchirurgische Klinik der Universität München, Klinikum Großhadern, München

\title{
Erfahrene Anästhesisten unverzichtbar
}

\section{Anästhesiologisches Management bei Implantation von Kardioverter/Defibrillatoren}

Das anästhesiologische Management bei der Versorgung mit implantierbaren Kardioverter/Defibrillatoren (ICD) hat sich in den letzten 20 Jahren grundlegend geändert. Die erste Defibrillatorgeneration (1981 bis 1989) wurde mithilfe der Thorakotomie implantiert. Die Defibrillation erfolgte über intrathorakale perikardiale „Patch“-Elektroden. Die Patienten wurden ausnahmslos in Allgemeinanästhesie versorgt und mit invasivem Monitoring (Arterie, zentralem Venenkatheter) überwacht. Patienten mit extrem reduzierter kardialer Funktion wurden darüber hinaus regelhaft mithilfe eines SwanGanz-Katheters überwacht. Damals war v. a. die häufige Testung der Defibrillationsschwelle ein Problem, da die myokardiale Pumpfunktion mit jeder Testung weiter beeinträchtigt wurde. Symptomatisch zu der Situation damals soll der Titel einer Veröffentlichung zu dieser Zeit genannt sein: „Hemodynamic deterioration during ICD implant“ [6].

Im Jahr 2010 ist eine völlig andere Ausgangssituation gegeben. Seit 1993 gibt es transvenös-pektorale ICD-Systeme, die nahezu gleich wie Herzschrittmacher implantiert werden. Patch- oder „Array“Elektroden müssen nur noch in Ausnahmesituationen verwendet werden. Die Aggregate agieren meist selbst als Elektrode („active can“), sodass lediglich eine weitere transvenös platzierte Elektrode in den rechten Ventrikel sowie ggf. eine in den rechten Vorhof gelegt werden muss.
Bei Herzinsuffizienz und Asynchronizität der Herzkammern werden häufig biventrikuläre Systeme eingesetzt, die dann zusätzlich noch eine Elektrode im Bereich des linksventrikulären Myokards (im Regelfall über den Sinus coronarius) erfordern. Neu entwickelte, rein subkutan implantierbare ICD-Systeme werden Teil des raschen Fortschritts sein.

Sellmann et al. ${ }^{1}$ zeigen in ihrer Übersichtsarbeit die heute gültigen Richtlinien für die Implantation der Kardioverter/Defibrillatoren auf. Eine Vielzahl an Studien konnte die Indikation extrem erweitern. Es wurden zahlreiche Gruppen von Patienten identifiziert, die von der Implantation eines ICD profitieren. Es soll hier nur exemplarisch die Gruppe der Patienten mit ischämischer Kardiomyopathie genannt werden $[4,5]$, die bei bereits eingeschränkter linksventrikulärer Funktion für die Primärprävention eines plötzlichen Herztodes identifiziert werden konnte.

Im zweiten Teil des Übersichtsbeitrags stellen die Autoren die verschiedenen Anästhesieverfahren gegenüber und zeigen auch die pharmakologischen Besonderheiten der häufig eingesetzten Medikamente auf. Die Aufrechterhaltung eines stabilen, adäquaten Analgosedierungsniveaus ist bei multimorbiden Patienten,

\footnotetext{
1 Sellmann T, Winterhalter M, Herold U, Kienbaum P (2010) Implantation von KardioverterDefibrillatoren. Wie viel Anästhesie ist nötig? Anaesthesist 59, S. 507-518
}

sehr häufig mit ausgeprägter pulmonaler Vorerkrankung, ein schmaler Grat und erfordert ständige Dosisanpassung und adäquate Titration in kleinen Schritten der einzelnen Substanzen durch den erfahrenen Anästhesisten.

\section{Kritische Anmerkungen zum Einsatz von Propofol}

Ergänzend soll hier noch ein kritischer Kommentar zum Propofol erlaubt sein. Diese Substanz ist wegen der fehlenden emetischen Wirkung, der subjektiv angenehmen Aufwachreaktion und der guten Steuerbarkeit sehr beliebt. Demgegenüber ist jedoch die negative Wirkung auf das Herz-Kreislauf-System schon seit langer Zeit bekannt. In einer Untersuchung an herzchirurgischen Patienten musste gerade die Gruppe mit koronarer Herzerkrankung und Propofol bereits nach dem sechsten Patienten vorzeitig terminiert werden [2]. Vor der Gabe von Propofol bei Patienten zur Implantation von Kardioverter/Defibrillatoren möchten die Autoren hier eher warnen. Es gibt - wie Sellmann et al. auch ausführlich beschreiben - weitaus bessere Medikamente, zumindest wenn die hämodynamische Stabilität der primäre Fokus ist.

Eine ganz entscheidende Frage ist jedoch die Notwendigkeit der intraoperativen Funktionstestung [7]. Während in der Literatur die intraoperative Testung der Defibrillationsschwelle weiter kon- 
trovers diskutiert wird $[1,3]$, verzichten bereits viele Implantationszentren in der Praxis darauf. Neue Verfahren, wie die Abgabe von Testschocks ohne vorherige Induktion von Kammerflimmern, werden in Zukunft die Prozedur weiter vereinfachen. Dadurch werden sich auch die Anforderungen an das anästhesiologische Management erheblich ändern. Gerade die Testung der Defibrillationsschwelle verursachte bei vielen dieser schwer kranken Patienten Herz-Kreislauf-Probleme [6]. Ohne die Notwendigkeit der Testung bei der Implantation lassen sich die meisten Eingriffe in Lokalanästhesie und Analgosedierung durchführen.

Die invasive Blutdruckmessung und v. a. jedoch die Anwesenheit eines in der Betreuung dieser multimorbiden Patienten erfahrenen Anästhesisten bleiben hingegen wegen der Vielzahl möglicher, schwerwiegender perioperativer Komplikationen (wie z. B. Pneumothorax, Hämatothorax, Perikardtamponade, Induktion maligner Arrhythmien etc.) obligat. Die Autoren können Sellmann et al. in folgendem Statement nur beipflichten:

„Die Durchführung einer Analgosedierung und einer Anästhesie für die Aggregattestung ausschließlich durch einen Internisten oder Herzchirurgen stell nach Meinung der Autoren im Schadensfall eine vermeidbare Gefährdung und somit ein Organisationsverschulden dar."

\section{Taugun}

P. Tassani-Prell

\section{Korrespondenzadresse}

Prof. Dr. P. Tassani-Prell

Institut für Anästhesiologie, Deutsches Herzzentrum München, Klinik für Anaesthesiologie der Technischen Universität Klinikum rechts der Isar Lazarettstraße 36, 80636 München tassani@dhm.mhn.de

Interessenkonflikt. Der korrespondierende Autor gibt an, dass kein Interessenkonflikt besteht.

\section{Literatur}

1. Blatt JA, Poole JE, Johnson GW et al (2008) No benefit from defibrillation threshold testing in the SCD-HeFT (Sudden Cardiac Death in Heart Failure Trial). J Am Coll Cardiol 52:551-556

2. Haessler R, Madler C, Klasing S et al (1992) Propofol/fentanyl versus etomidate/fentanyl for the induction of anesthesia in patients with aortic insufficiency and coronary artery disease. Cardiothorac Vasc Anesth 6(2):173-180

3. Hall B, Jeevanantham V, Levine E et al (2007) Comparison of outcomes in patients undergoing defibrillation threshold testing at the time of implantable cardioverter-defibrillator implantation versus no defibrillation threshold testing. Cardiol J 14:463-469

4. Moss AJ, Hall WJ, Cannom DS et al (1996) Improved survival with an implanted defibrillator in patients with coronary disease at high risk for ventricular arrhythmia. Multicenter Automatic Defibrillator Implantation Trial Investigators. N Engl J Med 335(26):1933-1940

5. Moss AJ, Zareba W, Hall WJ et al; Multicenter Automatic Defibrillator Implantation Trial II Investigators (2002) Prophylactic implantation of a defibrillator in patients with myocardial infarction and reduced ejection fraction. N Engl J Med 346(12):877-883

6. Steinbeck G, Dorwarth U, Mattke S, et al (1994) Hemodynamic deterioration during ICD implant. Predictors of high risk patients. Am Heart J 127:1064-1067

7. Swerdlow CD, Russo AM, Degroot PJ (2007) The dilemma of ICD implant testing. Pacing Clin Electrophysiol 30: 675-700

\section{Hübler, T. Koch \\ Komplikationen in der Anästhesie}

Heidelberg: Springer-Verlag 2010, 1., 355 S., (ISBN 978-3-642-01041-5), 39.95 EUR

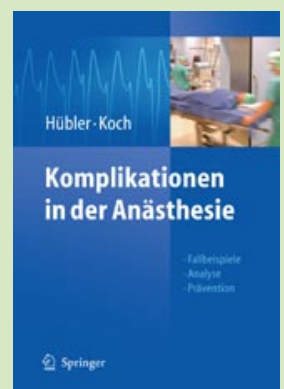

Das Einzige, was an diesem Buch herkömmlichen Vorstellungen entspricht, ist der lapidare Titel!

Über ",Komplikationen in der Anästhesie" wurden schon einige deutsch- und englischsprachige -,

fachlich sicher kompetente Bücher geschrieben. Das, was die beiden Autoren Matthias Hübler und Thea Koch aber hier mit diesem Buch präsentieren, liest sich wie ein unglaublich spannend geschriebener Actionroman, der den Leser unvermittelt mit realitätsnaher Dramatik in die OP's und Aufwachräume versetzt. Da glaubt der Leser, selbst am Ort des Geschehens zu sein, mitzudenken, mitzufühlen und manchmal mitzuleiden. Anhand von 30 Fallbeispielen eigentlich 30 Kurzgeschichten, eine spannender als die andere -, die sich alle irgendwann am Dresdner Universitätsklinikum, zumindest virtuell, zugetragen haben, werden praktisch alle relevanten Fehler und Komplikationen, mit denen wir tagtäglich konfrontiert sind, mit evidenzbasiertem Wissen, anerkannten Algorithmen und sinnvollen Interventionspfaden unterlegt und zu einem spannenden Ganzen verwoben.

Gekonnt vermeiden die Autoren, mit erhobenem strafenden Zeigefinger Fehler aufzuzeigen oder belehrendes Besserwissen zu vermitteln. Hier wird motivierende offene Fehlerkultur überzeugend dargestellt, manchmal sogar unterstützt durch den dramaturgischen Kunstgriff köstlicher Ironie und Selbstironie. Und es bleibt auch Raum für Gedanken zur Lösung zwischenmenschlicher Konflikte im nervenaufreibenden Klinikalltag.

Geprägt wird das Konzept dieses Buches sicherlich durch den inzwischen reichlichen Erfahrungsschatz mit dem Dresdner "Critical Incident Reporting System", für das Thea Koch und Matthias Hübler seit einigen Jahren zeichnen.

Insgesamt ist den Autoren nicht nur ein exzellent geschriebenes Buch über Fehler und Fehlerkultur im anästhesiologischen Klinikalltag gelungen, sondern darüber hinaus ein dramaturgisches Meisterwerk im Sinne einer für uns alle spannenden „Must have" Lektüre.

Professor Helfried Metzler (Graz) 